\title{
THALAMIC HYPERTROPHY OR GLIOMATOSIS OF THE OPTIC THALAMUS
}

\author{
BY \\ S. NEVIN \\ From the Hospital for Nervous Diseases, Maida Vale \\ (RECEIVED 29TH July, 1938) \\ Introduction
}

IT is well recognized that in the optic nerves and brain stem tumours of the glioma group frequently give rise to a general enlargement of the area involved, rather than a localized tumour formation. In such cases the new growth is diffuse and infiltrating, and there is considerable variation in the size, shape, and type of the tumour cells, so that classification of these tumours on an embryological basis has proved difficult. Similar types of growth may involve the optic thalamus. Here, however, they have been less frequently studied, although diffuse glioma of the brain stem generally extends to some degree into the optic thalamus. It is possible that cases in which the thalamus is predominantly involved are not common, and it is therefore proposed in this paper to describe three such cases, and to discuss their pathology and relationship to gliomas in general. The appropriate literature will be referred to after the general discussion.

\section{Case 1}

Clinical History.-P.S., a girl aged seven, was admitted to the Hospital for Nervous Diseases, Maida Vale, on the 25th October, 1935, under Dr. Feiling. She died on the 19th November, 1935.

The family history was negative. The patient was born with café-au-lait spots on her hip and back. She went to school at five years of age, but made no progress, and was clearly not as intelligent as her brothers and sisters. She was well up to ten months before her death, when she became quiet, played less with other children, and began to lose weight. She also began to have vacant turns, and four months later she complained of headaches and loss of vision in the left eye. The headaches increased in severity, and after admission to hospital they were very continuous and frequently associated with vomiting.

On examination she was very thin and wasted, drowsy and inco-operative. The left eye was blind, and the optic disc showed primary optic atrophy. Vision in the right eye appeared to be normal ; the visual field was full to rough tests and the optic disc was normal. The right pupil reacted well to light, but the left only very slightly. There was slight weakness of the left sixth nerve. The musculature generally was thin and wasted, but no alteration in motor power, reflexes, or sensation was observed 
X-ray of the skull showed slight downward displacement of the anterior clinoid processes, especially the left, which was also partially eroded. At operation a large hypothalamic tumour was found, the greater part of which was removed, but the patient died the next day.

POST-MORTEM EXAMINATION.-On removal of the brain the hypothalamic region was found hollowed out by the tumour and the pituitary region of the skull flattened and thinned. The floor of the third ventricle forwards from the corpora mammillaria was destroyed and there was dilatation of the antero-inferior part of the third ventricle. Vertical section of the brain revealed that the thalami were paler and firmer than normal and slightly enlarged throughout their whole extent, but no other abnormality was observable with the naked eye (Fig. 1).

Microscopical Description.-The Hypothalamic Tumour.-This consisted of round, oval, and elongated nuclei with well-defined nuclear membrane and a granular

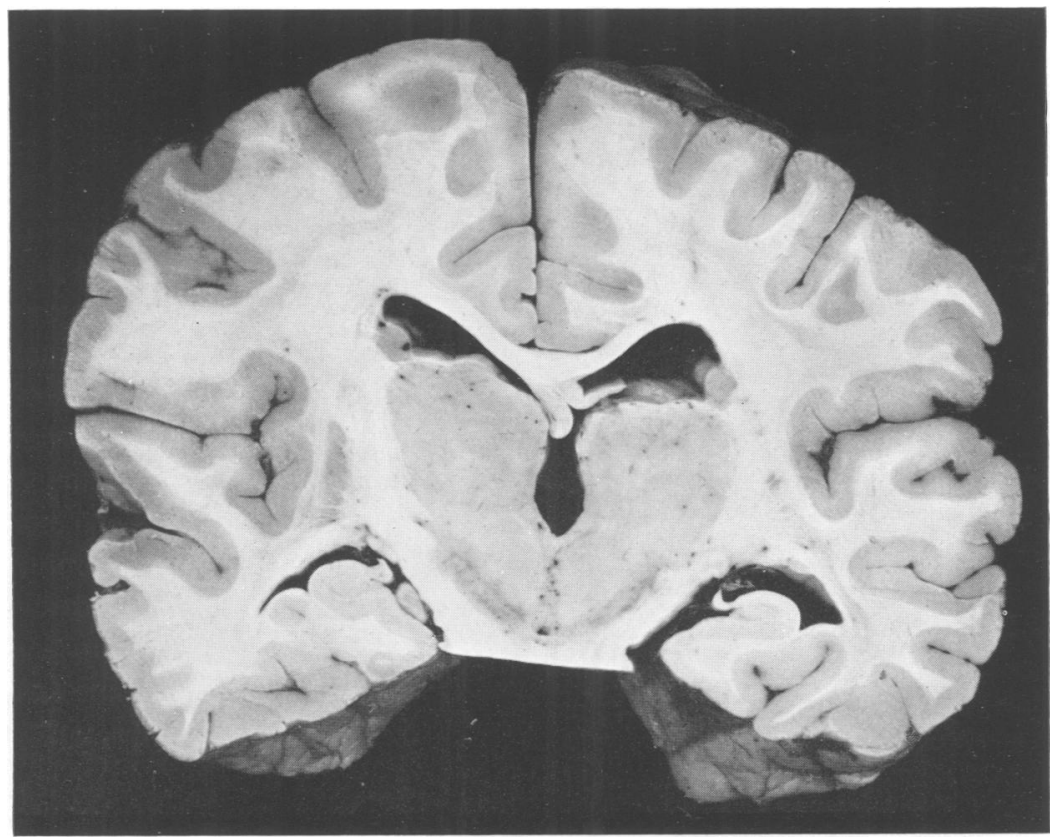

Fig. 1.-Case 1 : Vertical section of the brain, showing the slight bilateral enlargement of the thalamus.

chromatin network lying in a light fibrillar stroma (Fig. 2). No cell body was seen with ordinary nuclear staining. In parts the microscopic field was filled with loosely arranged nuclei varying very much in size and shape, while in other parts fibrillar bundles of elongated cells were found. There was dense cellular condensation along some blood vessels, and the irregular fibrous tissue coat of these vessels was infiltrated with tumour cells. Coarse, thick neuroglial fibres were everywhere present in the tumour. These could often be seen arising from bipolar or irregular shaped cells in Mallory or Victoria blue preparations, but there were also numerous rounded cells with no processes. These were especially numerous in the irregular masses around the blood vessels. The tumour may be designated a polar spongioblastoma, although this does not accurately describe its histogenesis. It is clearly very similar to the gliomas occurring in relation to the optic nerve, and it may possibly have arisen from the left nerve, as the optic chiasma showed a slight new formation of irregular shaped neuro- 
glial cells. It was localized to the hypothalamic region, and did not invade the optic thalamus or adjacent structures.

The Optic Thalamus.-On microscopical examination the thalamic hypertrophy was found to be due to a diffuse new growth of neuroglial cells, which was nowhere very intense and had not produced any definite changes in the essential nervous elements (Fig. 3). No cell body was seen with ordinary nuclear staining, but Hortega's silver carbonate method for astrocytes revealed that the cells varied in size, shape, and type from immature spongioblasts to enlarged and irregularly shaped astrocytes. In the spongioblasts the nucleus was for the most part small and round and heavily impregnated, and the cell body generally small, round, or oval with a coarse granular appearance and no very definite cell membrane. The cell body often tailed off into a single process, but frequently no process could be stained. Bipolar cells were rarely seen. In some cells with round nuclei the cell body was larger, and several rudimentary processes could be made out, although the general staining of the cell body was similar to that in the small unipolar spongioblasts. The irregular-shaped nuclei showed little cell body, but faint processes could be made out opposite the angulation

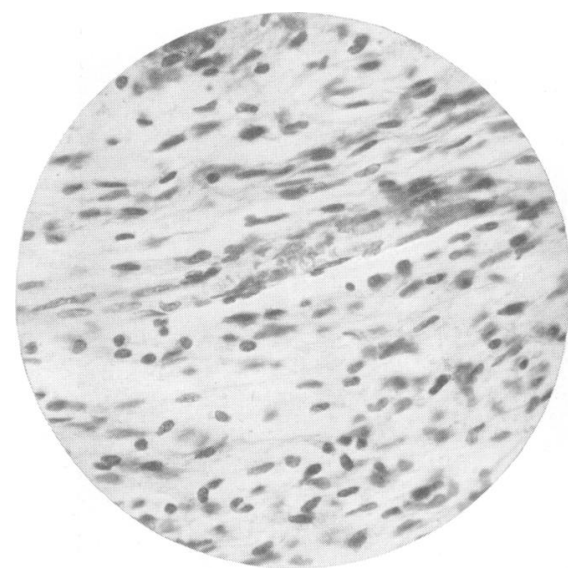

Fig. 2.-Case 1: Structure of the hypothalamic tumour. (Hæmatoxylin and eosin.)

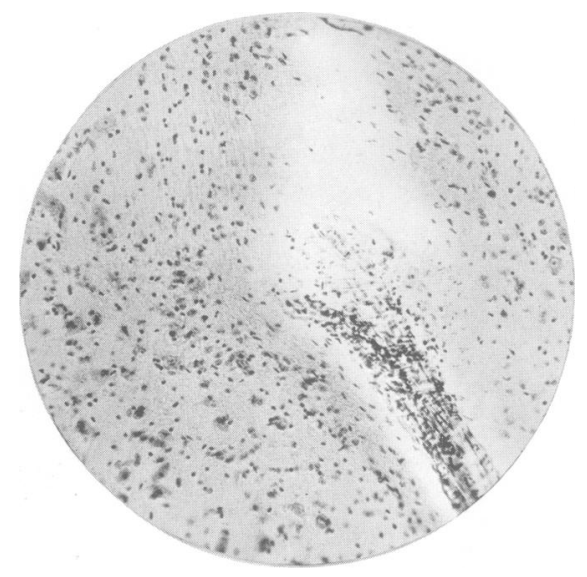

Fig. 3.-Case 1: The diffuse cellular overgrowth in the thalamus. (Iron hæmatoxylin and van Gieson.)

of the nuclei. Such cells were probably transition stages between the spongioblasts and the mature astrocytes. The latter varied very much in size; the nucleus was granular and lighter stained than in the spongioblasts, and the cell body was clear with a definite cell membrane and sharp though faintly stained cell processes.

The new-formed cells were scattered fairly evenly throughout the thalamus, and they also extended into the upper part of the midbrain. Frequently little clumps of three or more cells were found, especially around nerve cells. There was also some cellular accumulation around some blood vessels and underneath the superior and inferior surface of the thalamus and round the margin of the brain stem. This was associated with dense, fine gliosis which also extended irregularly throughout the thalamus and upper part of the midbrain as a loose network varying from point to point. That round the blood vessels consisted of closely packed piloid bundles of fibres running in the long axis of the vessel. This glial condensation displaced the myelinated fibres in a regular manner from the immediate proximity of the larger vessels. The increased marginal gliosis around the midbrain extended down the brain stem to the upper part of the spinal cord. It spread inwards irregularly in different regions and throughout the brain stem elongated and irregularly shaped neuroglial cells were everywhere present. There appeared also some increase of cells 
in the internal capsule, while in the lenticular nucleus and island of Reil the neuroglial nuclei were very irregular in size, shape, and form although there was no definite cellular increase. The actual limits of the cellular overgrowth in the thalamus were therefore difficult to define.

The cerebral cortex was not involved in the new growth, but in different areas there was increased marginal gliosis, which in some fissures was adherent to the walls of the blood vessels. In these sites recent hæmorrhage had occurred around the blood vessels and between them and the cortex.

Changes in the Essential Nervous Structures.-Myelin preparations showed no gross demyelinization in the thalamus or brain stem, and the internal capsule was darkly stained. Also in these areas the nerve cells were normal in appearance, and there was no definite alteration in their number or arrangement. In the giant celled precentral area of the cortex, however, many of the large pyramidal cells were very irregular in shape, and the protoplasm stained darkly and diffusely, showing little differentiation of Nissl granules. In some cases excessive branching of the dendrites close to the cell body occurred, and others were abnormally placed, the apical dendrite pointing towards the white matter, while the nucleus was also displaced to one or other side. These changes were present in numerous sections from both hemispheres. Their pathological significance is doubtful, but they may possibly indicate some slight developmental abnormality in regard to the cells. A detailed study, however, of the cerebral architectonics was not made.

\section{Summary and Discussion of the Case}

The case was characterized clinically by a slight degree of mental deficiency. skin pigmentation as in Von Recklinghausen's disease, and for ten months before her death the signs and symptoms of a hypothalamic tumour. Pathologically, as well as this tumour there was present a symmetrical hypertrophy of the thalamus. This resulted from a diffuse overgrowth of spongioblastic and astrocytic cells very variable in size, shape, and type throughout the thalamus, and extending also into the upper part of the midbrain. This new growth of cells was clearly of blastomatous origin, but it did not admit of classification as a single type of tumour formation. It is unlikely that a tumour could arise in one place and spread through the thalamus in such a way as to give rise to dense perivascular gliosis and cellular accumulations round the nerve cells. The varied form and differentiation of the cells would also require two or more classifications of the type of growth. The cellular proliferation appears to have developed locally over a wide area, and may therefore be designated a gliomatosis of the optic thalamus. The presence of other abnormalities, some clearly developmental, such as the café-au-lait patches on the skin, the marginal gliosis in the cortex and the brain stem, and the hypothalamic tumour suggest the possibility that the growth may be congenitally predetermined. As a developmental abnormality neuroglial cells capable of giving rise to it may be present over a wide area. They may be mature or undifferentiated, but as differentiation of the multiplying cells seems a feature of the growth, the latter is more probable. The factors determining the origin and rapidity of the cellular proliferation are entirely obscure, which is also the case with regard to blastomatous malformations in general. 


\section{Case 2}

For this case I am indebted to Dr. Denny Brown and Dr. Greenfield, and the notes are from the routine clinical and pathological records of the National Hospital, Queen Square.

Clinical History.-A.B., a man aged 60 years, was admitted to the National Hospital on 8th March, 1937, under Dr. Denny Brown. He died on 31st March, 1937.

He had one younger brother, who was subject to fits, and his first wife, who came of an epileptic family, had two children, one of whom was epileptic.

Apart from a severe blow on the chin at the age of forty, the patient suffered no illness and was well up to six months before his admission to hospital, when he began to complain of headaches, drowsiness, and failure of memory. The onset of these symptoms was gradual, and they slowly became worse. The headaches were chiefly vertical and came in severe attacks of short duration. He was continually drowsy, and he tended to fall asleep several times in the day. The failure of memory was especially for recent events, and he would forget the day of the week and even which bedroom he occupied. Before admission to hospital he also complained of giddiness and general unsteadiness in walking.

On examination he was drowsy, but co-operative. His speech was slow, but otherwise normal. He was normally orientated in time and place. There was no papillœdema nor other abnormal sign in the cranial nerves. The motor system reflexes and sensation were all normal, but his gait was slow and slightly unsteady. The cerebrospinal fluid was under a pressure of $230 \mathrm{~mm}$. of water and was normal except that the total protein was $90 \mathrm{mgms}$. per 100 c.c. and the globulın tests were positive. Ventriculography by Mr. Jackson showed defective filling of the body of the right lateral ventricle and deviation of the ventricular system to the left. The patient gradually became worse and died, operative interference not being considered advisable.

Post-Mortem Examination.-The brain weighed 1,700 grammes. The medulla, pons, and right mammillary body appeared enlarged to the naked eye. Horizontal section revealed a uniform enlargement of the right optic thalamus, which bulged markedly into the third and right lateral ventricles (Fig. 4). The right thalamus was also paler and firmer than normal, except in the antero-medial part, which was softer than normal. The corpus striatum on the right side and the grey matter in the depths of the island of Reil were slightly paler than normal. The left hemisphere was normal in appearance, and the normal configuration of the brain was everywhere preserved.

Microscopical Examination.-This revealed that the naked eye changes were produced by a widespread cellular overgrowth which was most severe in the right optic thalamus, but also extended throughout the brain stem and the basal ganglia and adjacent areas of the right cerebral cortex. The microscopical features of this new growth will be described in the optic thalamus, and then its general distribution and variations will be followed.

In the thalamus nuclear stains revealed a diffuse and fairly regular cellular infiltration throughout the whole organ, which was, however, most dense antero-medially. The nuclei closely resembled normal neuroglial nuclei in general structure, but they varied greatly in size and shape. There were round, smoothly elongated, polygonal shaped, and irregularly lobulated forms, and the size varied from that of oligodendroglial nuclei to that of nerve-cell nuclei (Fig. 5). The polymorphous forms sometimes showed fine chromatin threads connecting two or three lobules, and dumb-bell and horse-shoe shaped forms also occurred. With Hortega's silver carbonate method for astrocytes the cells were found to be astrocytes and spongioblasts with a great variation in the form of the cell body corresponding to the variation in nuclear shape (Figs. 6, 7, and 8). The astrocytic cells showed a lightly staining and well-defined 
cell body and sharply outlined cell processes. Some were small, with hardly any cell body, and others very large, with coarse, thick processes which could be seen to extend long distances from the cell. Medium-sized forms were numerous ; many appeared elongated, others were triangular shaped, and some contained two nuclei. These cells formed only a part of the cellular overgrowth, and there were present numerous more immature cells. Some of these had a clearly outlined cell body, which was, however, faintly stained with no definite cell membrane and a few indefinite cell processes. In others, especially with very irregular shaped nuclei, only a little cell protoplasm but no processes could be stained, and in relation to some nuclei even this could not be made out. Some cells could, however, be labelled polar spongio-

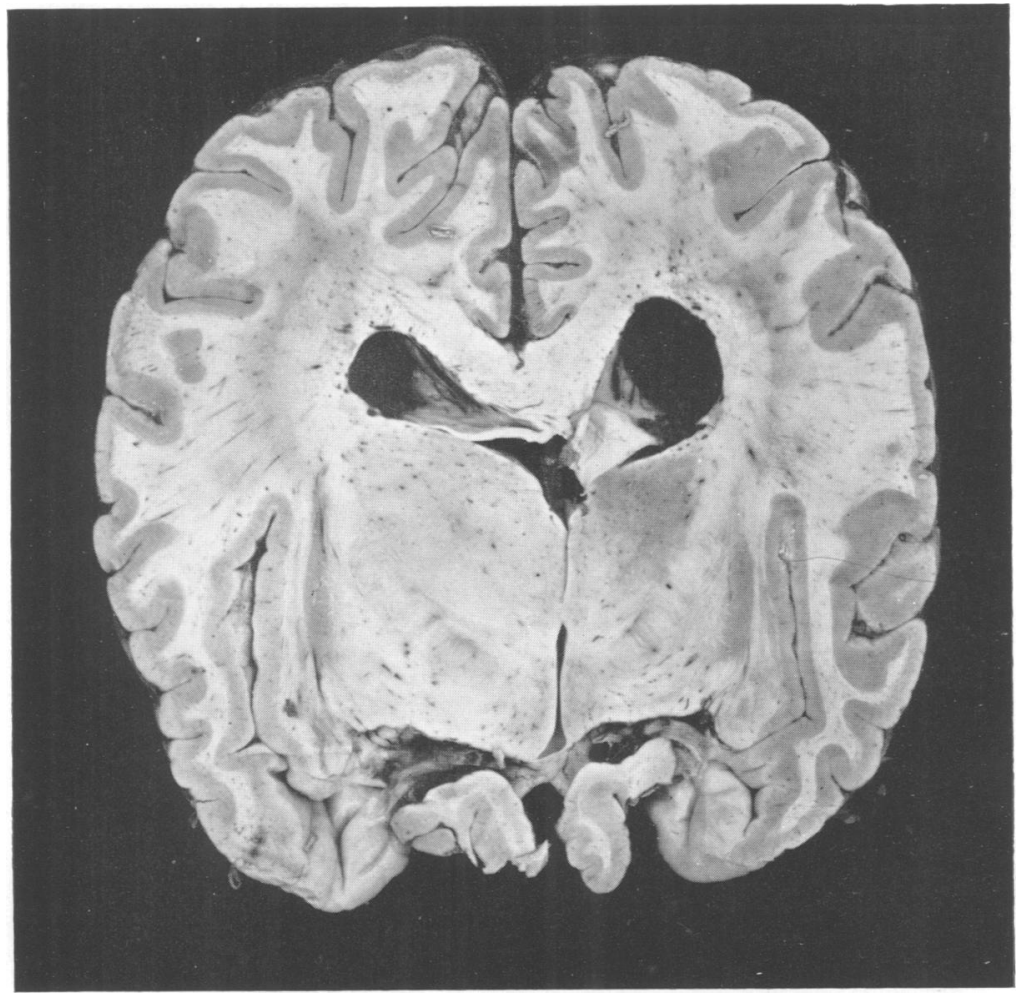

Fig. 4.-Case 2 : Horizontal section of the brain, showing the diffuse enlargement and pallor of the right thalamus and also the pallor and increased depth of the cortex in the island of Reil.

blasts because the nucleus was round or elongated, and a long process, faintly stained, could be seen passing away from one pole of the cell. These were small, but similar cells in which the bodies and processes were large and thick, and stained similarly to the giant astrocytes, were also present (Fig. 7). The processes of these, and also those from astrocytic cells, were frequently attached to vessel walls. Bipolar spongioblasts were rarely seen. The different types of cells were evenly mixed throughout the tissues, but little clumps occurred around nerve cells and frequently one or two astrocytic cells were grouped with several more immature cells.

In the posterior part of the thalamus the growth was moderately severe, and many astrocytic cells with round cell body and numerous processes arising right round the cell body were present. Some of these lay close to nerve cells, and then their processes enclosed the nerve cell as in a basket (Fig. 6). The processes of these cells 

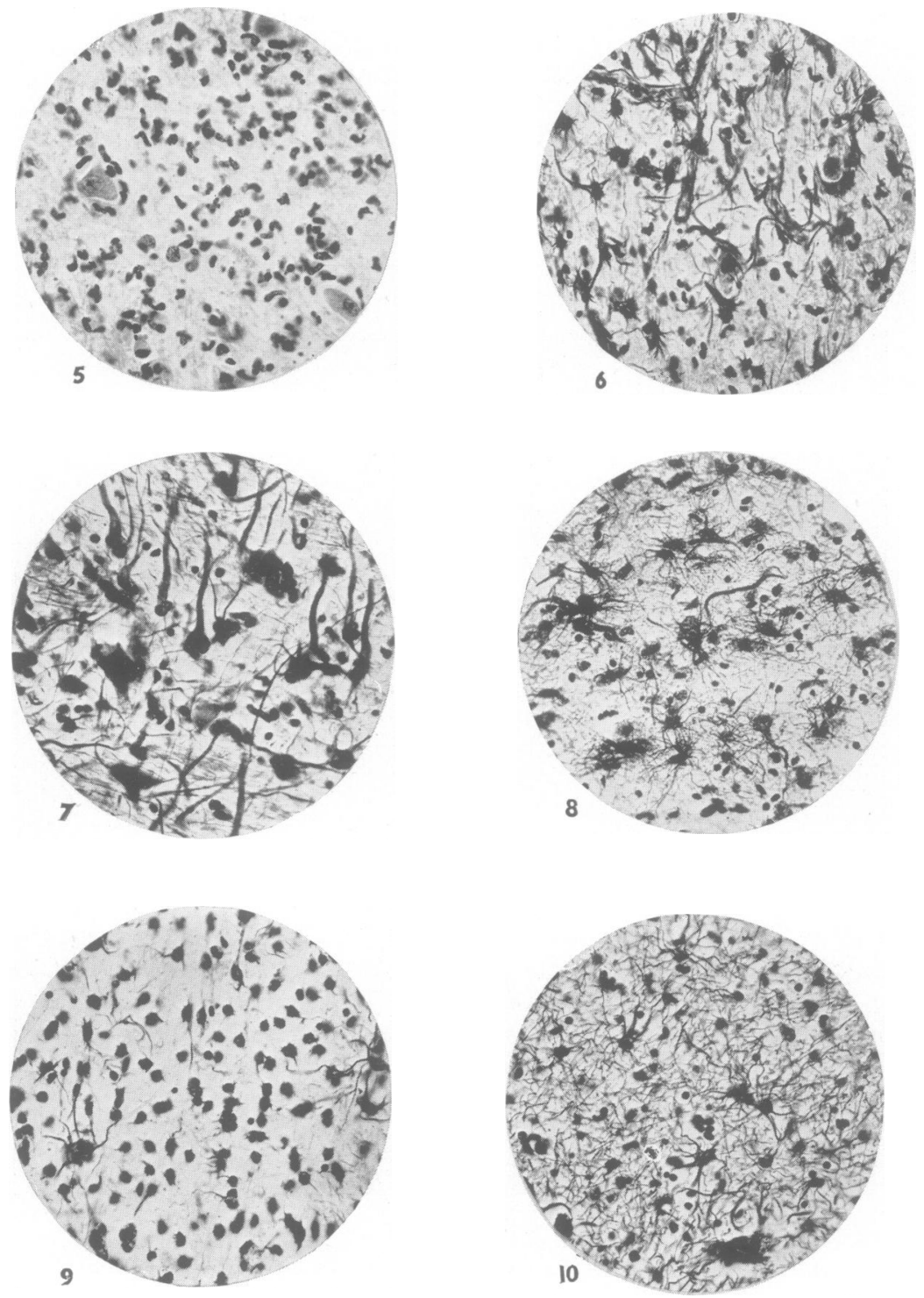

Fig. 5.-Case 2: The varied size and shape of the nuclei of the cellular overgrowth in the thalamus. (Iron hæmatoxylin and van Gieson.)

Fig. 6.-Case 2: The different cell types in the thalamus, and the processes of the blastomatous cells enclosing the nerve cells. (Hortega's silver carbonate method for astrocytes.)

Fig. 7.-Case 2: Another field showing predominantly large unipolar cells. (Hortega's S.C.A.) blue.)

Fig. 8.-Case 2: The diffuse glial overgrowth in the thalamus. (Anderson's Victoria

Fig. 9.-Case 2: The cortex of the island of Reil showing the new-formed cells with no fibre production in the background ; to be contrasted with Fig. 10. (Hortega's S.C.A.)

Fig. 10.-Case 2: The subcortical white matter of the island of Reil showing the fibrillar nature of the overgrowth. (Hortega's S.C.A.) 
were well stained with Victoria blue, and a fine glial network of varying density was present (Fig. 8). There was also glial condensation round the vascular spaces. In this area of the thalamus there was little alteration in the essential nervous structures compared with the degree of new cell formation. Degenerating nerve cells were sometimes seen, but there was no great reduction in their number. Those remaining contained large accumulations of lipoid staining dark red with Scharlach R. In the softened area of the anterior part of the thalamus the cellular overgrowth was very dense, and more immature cell forms were present and the neuroglial fibre production was slight. There was also much greater destruction of the essential nerve structures, although numerous myelinated fibres and nerve cells were still present. Scharlach $\mathbf{R}$ preparations showed much dark red lipoid scattered throughout the area, while myelin stains showed that the slight general pallor of the whole thalamus was accentuated in this region.

General Distribution of the Cellular Overgrowth.-The internal capsule showed no demyelinization, and in this structure the cellular overgrowth was much less, and the nuclei were for the most part round or elongated in the direction parallel to the fibres. In the caudate and anterior part of the lenticular nucleus the density of the cell proliferation corresponded to that in the posterior part of the thalamus. There was, on the whole, less formation of fine glial fibres between the cells, except on the posteromedial part of the globus pallidus adjacent to the internal capsule. In the anteroinferior part of the globus pallidus there was a small oval area of complete demyelinization, and in this the cellular overgrowth was dense and the cells were astrocytic with swollen cell bodies similar to gemastete cells. There were other immature cells present in the focus which were not swollen. In the anterior commissure the cellular overgrowth was not severe, but it gave rise to a fine gliosis which tended to be arranged parallel to the direction of the fibres, and this contrasted strongly with the appearance in adjacent parts of the caudate and lenticular nucleus, where the round astrocytes with numerous processes did not seem to give rise to a great glial fibre formation throughout the tissues.

The new growth in the right hemisphere also involved the depths of the island of Reil, the antero-medial part of the temporal lobe, the anterior part of the corpus callosum and adjacent white matter, and the medial surface of the hemisphere.

In the island of Reil it was greater in the cortex than in the subjacent white matter, and was of such a degree that it led to gross destruction of nerve cells so that the cell layers were not recognizable; also the residual nerve cells were filled with lipoid. The new-formed cells were, for the most part, mature astrocytes of medium size with sharply stained cell processes, but they did not give rise to a glial network in the background (Fig. 9). In the subjacent white matter, although the cellularity was much less, a glial network was present (Fig. 10). The involvement of the frontal lobe was most marked immediately underneath the ventricular surface, where a subependymal overgrowth of bipolar spongioblasts and round or oval cell forms was found. These cells extended considerable distances into the subjacent white matter and large condensations were present around numerous blood vessels. Small areas of the adjacent cortex were also involved and here a slight cellular overgrowth extended right through the cortex to the pia arachnoid.

In the left optic thalamus and basal ganglia there was no definite cellular overgrowth but the neuroglial nuclei were in general larger and very much more irregular in size than normal.

The new growth extended throughout the whole extent of the brain stem and involved also the commencement of the spinal cord. Weigert Pal preparations of the brain stem showed no pallor or tract degeneration. It was in general greater on the right side, and was similar to that found in the thalamus and basal ganglia. In the pons it was greater dorsally than ventrally and in the pontine nuclei more than in the fibre bundles. It was everywhere accompanied by a network of glial overgrowth which varied very much in density. There was also increased marginal gliosis throughout 
the whole extent of the brain stem. The cerebellum did not show any definite abnormality, but in the region of the dentate nucleus there appeared to be much greater variation than normal in the size and shape of the neuroglial nuclei. It is recognized, however, that great variations have been described in the normal neuroglia in this region, so that the significance of this point is doubtful.

\section{Summary and Discussion of the Case}

The case was characterized clinically by a six months' history of headache, drowsiness, and failure of memory. Pathologically there was found a new growth of spongioblastic and astrocytic cells throughout wide areas of the central nervous system, predominantly affecting the right optic thalamus, but also the adjacent areas of the cerebral hemisphere and the brain stem. The overgrowth was superimposed upon the essential nervous elements, and had only led to severe alteration of these structures in the small area of greatest growth in the anterior part of the right thalamus. It was impossible to define clearly the limits of the growth, as slight changes extended widely from the main lesion, and the difficulty was to pronounce the neuroglia entirely normal in the uninvolved regions of the brain, as for example, in the dentate nucleus. With regard to the nature of the new growth, it seems impossible to classify it as a tumour formation which had arisen in some area and spread, either by fibre tracks or along vascular pathways or in some other manner widely throughout the nervous system. This would presuppose a remarkable ability for migration among normal tissues of individual tumour cells, and an ability to stop in special sites, as for example, around nerve cells and in the grey matter of the pons, which was more predominantly affected than the adjacent white matter. Moreover, the cells have not escaped entirely from the influence of the surrounding tissues, as is shown clearly by the different character of the cells in the grey and subcortical white matter of the island of Reil. Furthermore, differentiation of multiplying cells seems an essential feature of the cellular proliferation, because if such is not the case the regular arrangement throughout the affected area of cells in various stages of differentiation from spongioblasts to astrocytes is difficult to understand. For these reasons the pathological process has been considered a diffuse gliomatosis which has arisen in situ over a wide area, or having commenced in one area has affected adjacent areas by liberation of chemical substances or growth factors. On this basis the involvement of the temporal horns and the white and grey matter of the medial surface of the hemisphere is more easily understood. A developmental predisposition to the blastomatous change seems likely, although there is no direct evidence for this factor, apart from the abnormal variation in form of the neuroglial cells in parts of the uninvolved cortical areas. The origin of the growth, therefore, is probably from widespread abnormal neuroglial cells perhaps not fully differentiated, but the factors determining the late onset and rapidity of the growth of the proliferating cells are entirely obscure.

Case 3

Clinical History.-S.N., a married woman aged 46, was admitted to Maida Vale Hospital on 21st December, 1936, under Dr. Golla. She died on 1st January, 1937. 
The family history of the patient was negative. She herself was well up to nine months before admission to hospital. At this time, following her mother's death, she seemed to lose interest in things, became forgetful, and began to complain of headaches. The headaches, which at first occurred once or twice a week and lasted two or three hours, became more frequent and more severe, and were associated with drowsiness and at times disorientation and confusion. These symptoms gradually progressed and she became listless and dreamy and unable to take care of herself.

ON EXAMINATION.- She was drowsy and inco-operative, and it was difficult to hold her attention. She understood simple commands, knew where she was, and recognized her friends. Her memory was vague for recent events and her speech was very slow. She was also incontinent of urine and fæces. There was no papillodema and the cranial nerves were normal except that the pupil reaction to light was diminished in both eyes, especially the right. She lay in bed on her back with arms

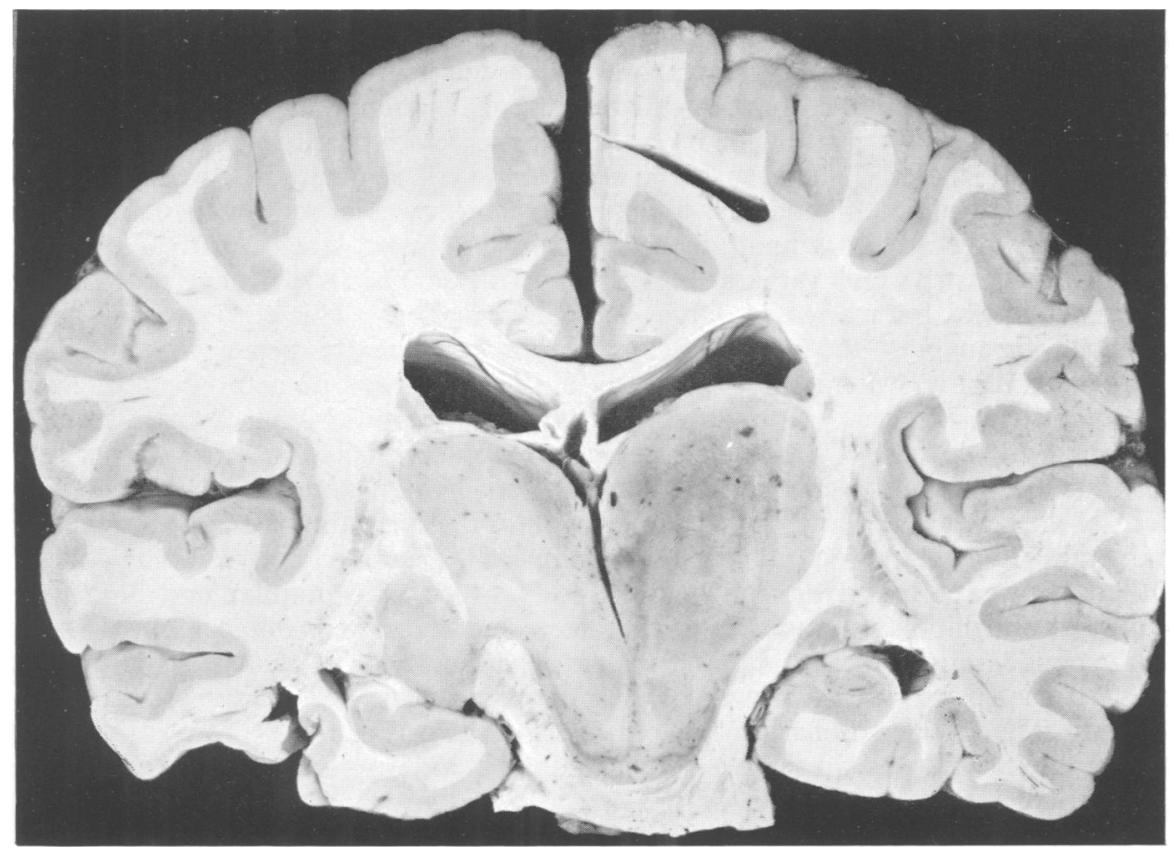

Fig. 11.-Case 3 : Vertical section of brain showing the enlargement of the optic thalami.

flexed and adducted and legs flexed. There was a generalized resistance to extension of the limbs. The rigidity seemed greater on the right side, and an irregular tremor was occasionally present in the right hand. No gross loss of power could be made out. Except that the left plantar was extensor and the left knee-jerk increased, the reflexes were normal. No definite sensory change was detected. The cerebrospinal fluid was normal, and the pressure was $175 \mathrm{~mm}$. water. The patient gradually became weaker, more drowsy, and one week after admission lapsed into unconsciousness, with stertorous breathing, loss of reflexes, and died in half an hour.

Post-MORTEM EXAMINATION.-Apart from a slight cerebellar pressure cone, there was nothing to note on the external surface of the brain. Vertical section revealed diffuse enlargement and discoloration of the optic thalami, especially on the left side, narrowing of the third ventricle, and moderate dilatation of both latcral ventricles and a linear area of cavitation in the medial part of the left cerebral hemisphere (Fig. 11). The left thalamus was diffusely enlarged throughout its whole 
extent, curving the internal capsule outwards, pushing the flattened third ventricle across the midline, and narrowing the body of the left lateral ventricle. The consistency of the whole organ was softer than normal, especially in its centre, which was also hæmorrhagic. The hæmorrhagic zone covered practically the whole transverse section of the thalamus at its centre, but decreased in extent anteriorly and posteriorly. The right thalamus was only slightly enlarged posteriorly. It was slightly paler than normal, but its consistency was not greatly altered. There was also an area of pallor inferior and lateral to the right thalamus and underneath the lenticular nucleus. It involved the tail of the caudate nucleus, the outer part of the internal capsule, and extended in an antero-posterior direction the whole length of the thalamus. The cavitation in the left hemisphere extended about two inches in an antero-posterior direction, was bridged over by the arachnoid, and the adjacent cerebral fissures extended into it. In its depths there was a thin lining membrane of indefinite structure, but towards the medial aspect of the hemisphere this was absent and the surface was more irregular. There was no surrounding gliosis and it was considered most probably the result of some developmental defect, but its pathogenesis was not clear.

Microscopical Description.-Microscopical examination revealed that the naked eye changes were produced by a diffuse new growth of neuroglial cells throughout both optic thalami. This varied in density and cell structure in different areas.

In the right optic thalamus the cellular overgrowth was of moderate intensity and composed of cells with round, oval, or irregular-shaped nuclei, with a granular chromatin network which varied very much in density. Their size in general resembled neuroglial nuclei, but many were larger and many smaller. This cellular proliferation extended throughout the whole thalamus, but was greater posteriorly and least marked anteriorly, where there was only a slight increase of neuroglial cells with irregular shaped nuclei. Hortega's silver carbonate method for astrocytes revealed that the cells were astrocytes and more immature neuroglial cells. The former were for the most part fairly large cells with a sharp but lightly stained cell body and welldefined processes. A few smaller astrocytes were present. Immature cells appeared more numerous than the astrocytes, and in many of these no cell body could be stained. Others showed a round or irregular cell body which was granular and ill-defined. Unipolar and bipolar spongioblasts were seen, but these were not numerous except in the condensation round the blood vessels, where bundles of bipolar and astrocytic cells associated with piloid masses of glial fibres were present. There was a general increase of neuroglial fibres throughout the thalamus (Fig. 12). This varied from point to point, and was always greatest around the blood vessels and in general towards the medial and inferior aspect of the organ. This new growth was superimposed upon the essential nervous elements of the thalamus, which had undergone little destruction compared with the intensity of the new cell formation. Some degenerating nerve cells, however, were seen, and those preserved showed large accumulations of fat in the cell body with distortion and irregularity of the neurofibril network.

In the left optic thalamus the growth was denser, but in general similar to that in the right optic thalamus. The nuclei were slightly larger and more irregular and there was greater variation in the size, shape, and form of the individual cells, many of which showed faint staining of the cell body with van Gieson and eosin stains. In the periphery the cell types were similar to those in the right thalamus, there being present numerous well-defined astrocytic cells and also more mature forms of moderate size. In the centre and hæmorrhagic areas the cells were greatly enlarged, and giant cells with long, coarse, thick processes of every possible shape and form were present (Fig. 13). Some were irregularly astrocytic, others triangular-shaped, and others bipolar. The cell bodies were clearly defined, but not so sharply outlined as those of the more regular-shaped astrocytes in the periphery. Some cells had two nuclei and there was considerable variation in the size of the cells with clearly outlined processes. There were other cells in which no processes were stained. Some of these had clearly defined and darkly stained cell body, but mostly the cell body was indefinite and 
irregular. Such cells varied much in size and form. Some had multiple nuclei, and occasionally they occurred in little clumps. There were also many round, oval, or elongated nuclei without any stainable cell body. In some areas the tumour cells were swollen, pale, and faintly stained, with the nucleus frequently compressed and flattened against the side of the cell. Such cells corresponded closely to gemastete
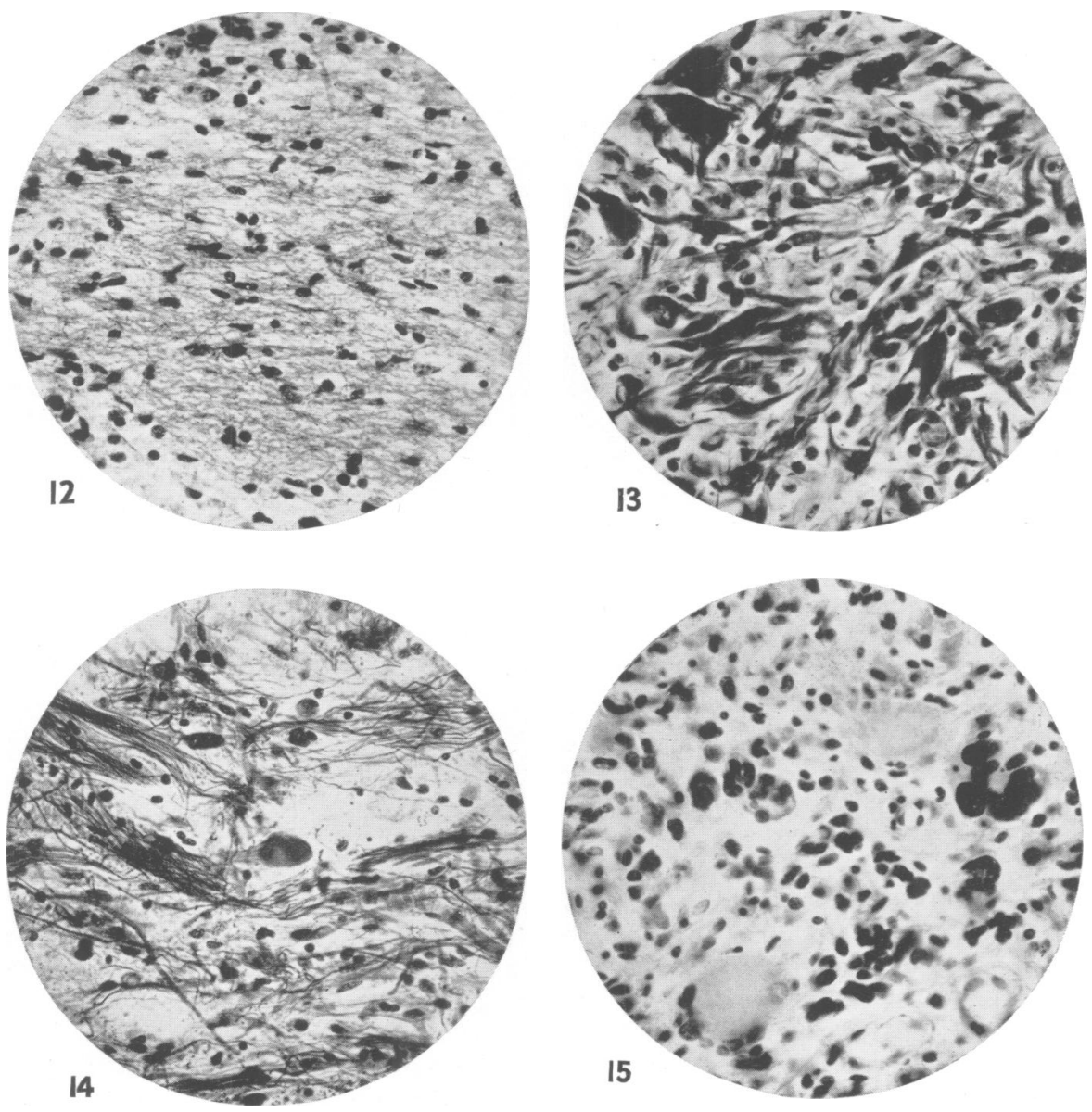

Fig. 12.-Case 3 : From the right thalamus, showing the general gliosis accompanying the growth. (Anderson's Victoria blue.)

Fig. 13.-Case 3: From the left thalamus, showing the giant nature of some of the cells and the coarse, thick processes which fill the background of the section. (Hortega's S.C.A).

Fig. 14.-Case 3 : From the left thalamus showing the bundles of glial fibres. (Anderson's Victoria blue.)

Fig. 15.-Case 3: The focus of tumour formation on the right side. (Iron hæmatoxylin and van Gieson.)

cells, being, however, much more irregular in size, shape, and form. There was a variable increase of fine glial fibres throughout the left thalamus. This consisted of a fine network. In some parts it was very slight, but in others it was dense and thick, and parallel bundles of fibres ran along the capillaries (Fig. 14). Foci of calcification were occasionally seen in relation to these vessels.

Although there was gross destruction of essential nervous elements, bundles and isolated myelinated fibres could be traced everywhere, even in the areas of greatest 
growth. Only a few nerve cells, however, remained towards the centre of the thalamus, and these were in various stages of degeneration. In myelin preparations the left thalamus was pale, but the internal capsule was darkly stained.

In the area of cellular overgrowth inferior and lateral to the right thalamus, a different appearance was found. Here the cells were closely packed together, forming a distinct tumour mass and completely replacing the normal tissues, leaving only a few scattered nerve fibres and cells round the periphery of the area. The cell nuclei were larger than those found even in the left thalamus, and giant lobulated nuclear masses occurred. Only the giant cells showed faint staining of the cell body with ordinary nuclear stains. With silver stains an irregular and indefinite cell body could be made out round many of the cells. Apart from a few isolated astrocytes no cell processes were stained except slight irregular blunt projections in some of the giant cells. Also there were no neuroglial fibres in the background. The blood vessels showed endothelial proliferation and degeneration of their walls and irregular mitoses were present. Although the cellular mass was sharply marked off from the surrounding tissues, the periphery of the growth was less dense and the marginal cells intermingled with those forming the diffuse overgrowth in the right thalamus. It was, however, distinctive from the diffuse growth found in the thalamus and from its microscopical features must be considered an area of tumour formation having the characteristics of a spongioblastoma multiforme (Fig. 15).

Changes in Other Parts of the Nervous System.-There was present in the upper part of the midbrain a general cellular overgrowth, greater dorsally. This was accompanied by much new glial fibre formation, but it had led to little disturbance in the essential nerve elements. Along the infero-lateral margin of the midbrain on the right side a wide band of increased marginal cellularity was present, comprised mostly of immature spongioblastic cells, but containing many astrocytic forms of varying size and shape. Throughout the remainder of the brain stem there was increased marginal gliosis, and in the dorsal part of the pons and medulla some increase of neuroglial cells showing a greater variation in size, shape, and type than is normally found. Many nerve cells in the brain stem and dentate nuclei showed accumulations of fat in the cell body, especially in the olivary nucleus. The lenticular nucleus was not generally involved in the overgrowth, but in this structure on both sides there was a general increase of neuroglial nuclei, very irregular in shape. The caudate nucleus was less definitely involved. There were very definite pathological changes in the medial aspect of the temporal lobes on both sides. This was greater on the right side, where a moderate cellular overgrowth, greater subpially, extended widely in the hippocampus and uncus. There was also dense marginal gliosis which extended forwards and was very severe round the anterior end of the choroidal fissure, where it involved the optic tract. On the left side, however, there was only slight increase of neuroglial nuclei in the white matter of the hippocampus and medial aspect of the temporal lobe and the marginal gliosis and cellularity round the anterior end of the choroidal fissure was less dense. There were no gross changes in the other parts of the cerebral hemispheres. In different areas foci of marginal gliosis were present and occasional subpial hæmorrhages. There was also irregular thickening of the meninges and small foci of endothelial cell proliferation. Many nerve cells also showed considerable fatty degeneration of the cell body.

\section{Summary and Discussion of the Case}

Clinically the case was characterized by a ten months' history of headaches, drowsiness, and such mental symptoms as failure of memory, confusion, and disorientation. Pathologically the predominant feature was the diffuse new growth throughout both optic thalami, consisting of a wide variety of abnormal neuroglial cells in various stages of differentiation superimposed upon the 
essential nervous elements and gradually replacing them. All stages were seen, from a slight new formation of small, regular-sized cells in the anterior part of the right thalamus with no alteration in the essential nervous elements, to a dense overgrowth of cells very variable in size, shape, and form replacing practically entirely the nerve cells and fibres in the centre of the left thalamus. Classification of the disease process as a single or even mixed cell glioma does not seem possible. The tumour would have to be considered a mixture of a diffuse astrocytoma, a gemastete cell astrocytoma, an astroblastoma, a polar spongioblastoma, and a spongioblastoma multiforme. The changes in the temporal horns might be considered a spread of the growth into those regions, but the slight pathological changes in the brain stem cannot be so considered, as it is inconceivable that the tumour cells could migrate so irregularly through the tissues from one point of origin.

Like the preceding cases, it must be considered as primarily a diffuse gliomatosis and of similar origin. Here, however, in the centre of the left thalamus there was such extreme rapidity of growth and variability of cell form that the differentiation from tumour formation was difficult. It perhaps could not be made if it were not that a gradual increase in severity of the pathological changes can be traced from other regions of the thalamus. This dense area, however, could be sharply distinguished from the focus of true neoplastic formation, which has developed in the area of growth lateral and inferior to the right thalamus. Here the cells had escaped entirely from the laws that govern normal growth of neuroglial cells, and were clearly growing as a focal tumour. They showed no processes and gave rise to no production of neuroglial fibres, and bore little resemblance to the abnormal neuroglial cells which were present in the diffuse overgrowth. One might regard them as a new race of cells, and the classification spongioblastoma multiforme or glioblastoma can be accurately applied to them. A few of the cells in the very dense areas of gliomatosis were similar to those in the tumour area, and it is difficult to assess their significance. They may indicate a gradual transition to the formation of a spongioblastoma multiforme, or they may be still under the control of normal tissues and capable of further differentiation.

The case, therefore, illustrates in itself different degrees of diffuse blastomatous overgrowth of the neuroglia, and the development in association with this overgrowth of a focus of anaplastic tumour formation. The factors determining the origin and nature of the diffuse growth are entirely obscure, as are also those determining the origin of the autonomous growth.

\section{General Discussion}

The three cases which have just been described have been classified as a diffuse gliomatosis of the optic thalamus.

In the first case the cellular proliferation was slow and very fibrillar, and had only led to slight enlargement of the thalamus. In the second case, however, it was more rapid, and a greater variety of abnormal neuroglial cells was present, and very dense new cell formation had occurred in the anterior part of the 
thalamus. A moderate gliosis varying in different regions accompanied the growth. In the third case the changes in the right thalamus corresponded closely to those in Case 1, while those in the left thalamus were very similar to those in Case 2, except that cells with large cell body and coarse, thick process were more numerous. An area of gemastete cell development leading to complete obliteration of all nervous structure was present in both. In the third case there had also developed an area of anaplastic tumour formation, so that all stages of blastomatous overgrowth of neuroglial cells were found in these three cases, from a slight overgrowth of practically normal cells right up to the focal formation of a malignant glioma. It seems probable, therefore, that in some cases other types of true tumour formation, e.g. an astrocytoma, an astroblastoma, or a polar spongioblastoma may arise on the basis of a primary diffuse gliomatosis, and may even become the predominant feature of the growth.

The pathological process in these cases is in general similar to that which has been described under the title of "Gliomatosis Cerebri" (Nevin, 1938). In some of the cerebral cases the thalamus was also involved in a diffuse fibrillar or cellular overgrowth, and a feature of the new cell formation in the cerebral cortex was the difference in the fibre production in the grey and the subcortical white matter. This was also present in the island of Reil in the second case of this paper, while other similar features were the perivascular overgrowth and the accumulation of new-formed cells round nerve cells. This pathological correspondence further emphasizes the suggestions which have been put forward with regard to the nature of the thalamic hypertrophy in the present cases, especially the possible developmental abnormality of the neuroglia which may form the basis for the new growth, as the cerebral cases were more closely related to Von Recklinghausen's disease. On the other hand, since each of the cases of gliomatosis of the optic thalamus in the present paper were associated to a greater or less extent with involvement of the brain stem in a manner similar to that found in pontine hypertrophy, emphasis is thereby given to the suggestion already made (Nevin, 1938), that these pontine growths and also gliomas of the optic nerve may possibly arise as a diffuse gliomatosis, and in future might profitably be studied from this angle.

\section{Literature}

There are comparatively few discussions of the pathological nature of gliomas of the optic thalamus in the literature. Weil (1933) has described a case of megalencephaly in a boy of six, associated with a diffuse, infiltrating tumour of the optic thalamus, brain stem, and cerebellum. The tumour cells had not led to great destruction of nerve tissue, and although there was some degeneration of myelinated fibres the nerve cells were well preserved. The tumour cells were condensed subpially in the spinal cord and cerebellum, and there were clusters of tumour cells around the Purkinje cells. The author considered the pathology of the cellular overgrowth to be a diffuse glioblastomatosis. The enlargement of the cerebral hemispheres was due to hypertrophy 
and not hyperplasia of the neuroglia, and in the areas of tumour growth the neuroglia had become first hyperplastic and later developed into a cellular glioblastomatosis.

Marburg (1906) described a somewhat similar case, except that the hypertrophy also affected the essential nervous elements, and the diffuse glioma was present especially in the thalamus and pons. It can hardly be doubted that in these cases the diffuse new growth has arisen on a developmental basis, and although there is possibly much variation in cell type and differentiation, the main pathological features of the growth are similar to those found in diffuse gliomatosis of the optic thalamus.

Stern (1937) has described a case in which a glioma of the optic nerve was associated with a diffuse tumour of the optic thalamus on the same side. The tumour cells in the thalamus were in the main bipolar spongioblasts, and she classified the case on an embryological basis as a spongioblastoma polare. She has kindly allowed me access to her preparations, and while the growth should be labelled a spongioblastoma polare if an embryological classification is attempted, its diffuse nature with the preservation of the essential nervous elements admit of the possibility that biologically it had arisen as a diffuse gliomatosis.

It is not suggested that all gliomas of the optic thalamus are in their essential nature a diffuse gliomatosis. Localized tumours of one or other type may arise. Some of these which have been studied, however, have shown a marked heterogeneity of cell structure. Baasch (1937) studied a glioma of the thalamus, aqueduct, and pineal region in a youth of seventeen. The cell picture in different places exhibited different stages of cell differentiation, and he considered the tumour to have arisen partly diffusely and partly in a circumscribed manner in an extensive subependymal embryonal layer. This possible pathogenesis is closely related to diffuse gliomatosis but in the latter condition the cells of origin, whether mature or undeveloped, must be considered to have migrated much farther from the position of the embryonic neural tube than the subependymal region. Baasch's case therefore supports the possibility that some more or less localized gliomas of the optic thalamus may arise on the primary basis of a diffuse gliomatosis. In any case, it is clear that all these diffuse growths in the thalamus must be studied not from the point of view of their classification according to the predominant type of cell found, but as a biological abnormality of the neuroglial system, and clues to their histogenesis may be found in the surrounding or even distant neuroglia.

Scherer (1937), in his study of gliomas of the cerebral hemispheres along these lines, has found an extraordinary variation in cell form in these growths, and he has considered that in some tumours there can be distinguished two sets of structures, the essential structure of the tumour and the secondary structures developed by the interaction of the neoplastic process and the invaded tissue. These secondary structures are such features as the clumping of tumour cells around nerve cells, along the vessels underneath the pia, and the gradual infiltration of the cortex. He can trace in some tumours from the periphery to the centre a gradual increase in new cell formation with increasing cell de-differ- 
entiation till in the centre of the tumour its essential structure is found having little resemblance to neuroglial cells. These secondary structures in Scherer's sense are features which characterize diffuse gliomatosis whether in the thalamus or in the cortex, so that the study of the essential nature of diffuse new growths in the nervous system is of importance from the point of view of gliomas in general. It seems unlikely that a tumour cell once having escaped entirely from the laws that govern normal growth could even by its interaction with normal tissue produce cells more akin to normal neuroglial cells. This may take place, but the possibility exists that growths showing marked secondary features in Scherer's sense may arise as a diffuse gliomatosis with anaplastic tumour formation predominating at the time of death. The other possibility exists that once a malignant glioma has developed, chemical substances liberated therein may diffuse into the surrounding tissue and cause a blastomatous reaction locally in the adjacent neuroglia, which would then be considered not as an interaction of spreading tumour cells and normal tissues, but as a blastomatous reaction of the normal neuroglia to a malignant growth. These observations only emphasize the point already made in reference to the optic thalamus that all gliomas must be studied as biological abnormalities of the neuroglia, and not simply with a view to classifying them as tumours arising from one or other type of embryonic cell.

\section{Summary}

Three cases of diffuse gliomatosis of the optic thalamus have been described. The histogenesis of this type of new growth and its possible relationship to the development of gliomas in general have been discussed.

This work was carried out during the tenure of the Pinsent Darwin Studentship in Mental Pathology.

I wish to thank the physicians who have allowed me to publish their cases. My thanks are also due to Professor Pulvertaft for permission to take the microphotographs.

\section{REFERENCES}

Baasch, E. (1937). Schweiz. Arch. Neurol. Psychiat., 39, 26.

Marburg, O. (1906). Arb. neurol. Inst. Univ. Wien, 13, 288.

Nevin, S. (1938). Brain, 61, 170.

Scherer, H. J. (1937). 2nd Congrès International Contre le Cancer. Brussels. 1936. Travaux Scientifiques, 2, 250.

Stern, R. O. (1937). Proc. roy. Soc. Med., 30, $1,096$.

Weil, A. (1933). Arch. Neurol. Psychiat. Chicago, 30, 795. 